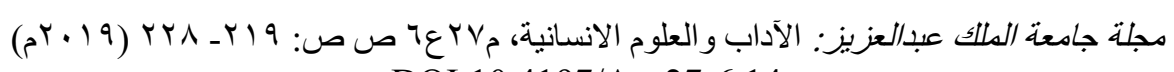

DOI:10.4197/Art.27-6.14

\title{
التاريخ والمؤسسة السياسية
}

إعداد الطالب: عبدالهادي نايف السلمي

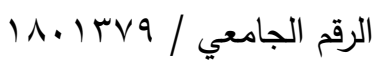

جامعة الملك عبدالعزيز بجدة

كلية الآداب والعلوم الإنسانية

قسم التاريخ - برنامج الدكتوراه في التاريخ الحديث

مستخلص. يشكل التاريخ مدخلاً مهماً في المؤسسة السياسة، وما يتعلق فيما تقوم به تلك المؤسسة من أدوار ، حيث تهيث يعد رافداً تستعين به المؤسسة السياسية تجاه ما يقابلها من تحديات، وما تبتغيه من توجهات. وقد جاءت هذه الدراسة لبحث تلك العلاقة بين التاريخ والسياسة ودوره في المؤسسة السياسية، وفي تحول المؤرخ من باحث سياسي إلى صانع في المعترك السياسي. وقد استخدم الباحث المنهج التاريخي لملاعمته لمثل هذا النوع من الدراسات، وقد توصل إلى أن التاريخ يستخدم

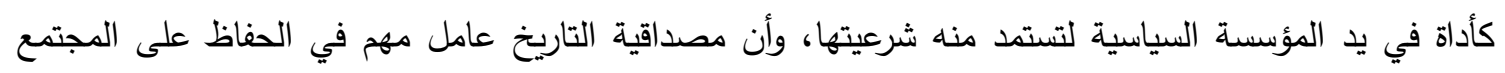
تحت مظلة المؤسسة السياسية، وأن ذلك لا يتحقق دون توفر مناخ عالي من الحرية للمؤرخين في تتاولهم للتاريخ. كما أكد الباحث على أهمية توفير المجال المنهجي المتاح للمؤرخ، مع ضرورة تطبيق أساليب البحث العلمي، والاهتمام بفهم كامل للمنظور التاريخي لدى دارسي التاريخ.

أو على نقيض ذلك مما تعرضت لله المجتمعات و البلدان من صراعات و كوارث كان محل اهتمام منذ القدم لأفراد من هذه المجتمعات، وتلك لرصد وتدوين تلك الفترات الزمنية و تأثيراتها على حياتهم مع هـه

\section{المقدمة}

إن حياة البشر ، و ما يعتريها من تغيرات عبر الحقب الزمنية المختلفة في مجالات عدة دينية و سياسية و اقتصادية، وما شملته من مظاهر ازدهار و نهضة 
ودوره في استخدام الحقائق وتوظيف التفاسير، كما ستتناول الدراسة دور التاريخ في صنع السياسة، وفي تحول المؤرخ من باحث سياسي إلى صانع في المعترك السياسي. علاقة التاريخ بالسياسة

لقد برز منذ القدم العلاقة القوية والمستمرة بين علم التاريخ وعلم السياسة وتكاد تكون هي الصورة الأوضح لالتقاء العلوم والتأثير المتبادل فيما بينها. لذلك لا يُستغرب اهتمام الكثير من الساسة على مر لإهاء العصور بدراسة التاريخ واستخدامه كرافد مهم من من لهن أجل نجاحهم. إن السياسيون يستخدمون التاريخ بطرق عديدة، فهم

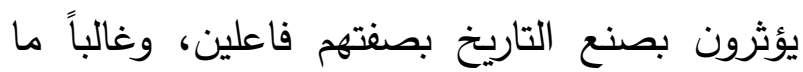
يكتبون التاريخ عن طريق ذكرياتهم ومذكراتهم .(Clark,2010,P1)

إن العلاقة بين السياسة والتاريخ تختلف باختلاف المجتمعات وتتغير على حسب نظام الحكم حيث تتناوب هذه العلاقة بين الإسهام الفعلي الحقيقي

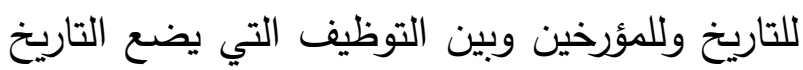
تحت خانة الوصاية لتحقيق مكاسب (كاستخدام النظام الإيراني للمظلومية التاريخية لآل البيت والشيعة كمثال)، أو لإعطاء شرعية تاريخية شعبية

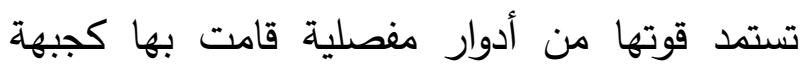
التحرير الوطني الجزائرية ونضالها ضد المستعدر

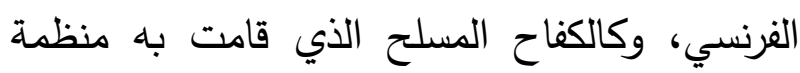
التحرير الفلسطينية (PLO).
محاولة دراسة الأسباب، و العوامل المحيطة بأي حدث، و ما العبر التي يمكن استتباطها من تلك دلك

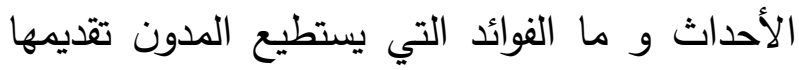

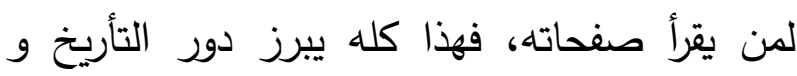
المؤرخ الذي يحاول أن يعطي الفرصة للأجيال المعاصرة والقادمة كي تتجول في أزمنة سابقة من لهن خلا أوراق سجلت و دونت أحداثها لكي تتضح الصورة أكثر بما يتعلق بالحاضر و المستقبل .

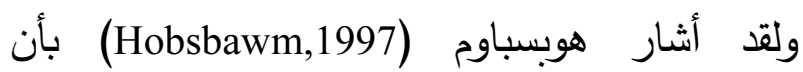
الماضي يُستخدم بدرجة كبيرة على كل مستويات المجتمع لفهم الحاضر وهذه حقيقة بديهية، والواقع أن الماضي شكّل الإطار المرجعي الذي عن طريقه

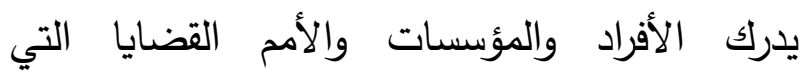
تواجه8م. ويتضح من ذلك مدى تتاول المؤرخ لجوانب كثيرة من حياة البشر وكيف أصبح هناك تلاقي وتداخل مع كثير من العلوم الإنسانية ومع المجالات الفكرية المتعددة والجوانب الاقتصادية.

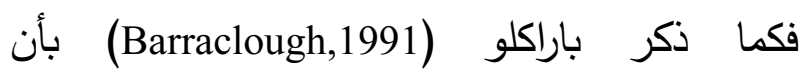
الاقتصاد هو أحد المجالات التي كان للتحليل التاريخي أثر كبير يعتمد عليه، حيث أدى التأكيد

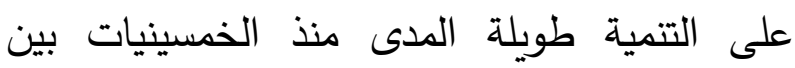
الاقتصاديين إلى زيادة أهمية البعد التاريخي في مئه تطوير نماذج افتراضية استقرائية. ومن هذا المنطلق سيتناول الباحث في هذه الدراسة ما يتعلق بعلاقة التاريخ بالسياسة والمؤسسة السياسة، 
ومن المقبول تماماً أن الأمم الحديثة التي تعتمد على وتفاعلها معها، وحالات الالتقاء والتتافر التي تحصل

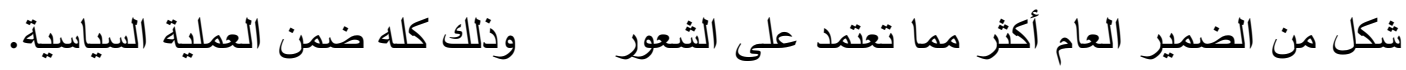

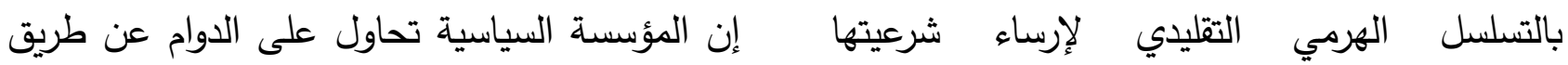
التاريخ إبراز آثار بلدها وتراثه وفنونه الثعبية،

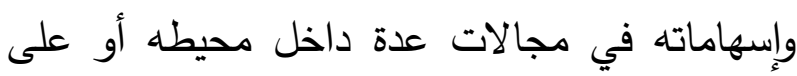
المستوى العالمي، وذلك لأسباب مهمة كرفع الروح

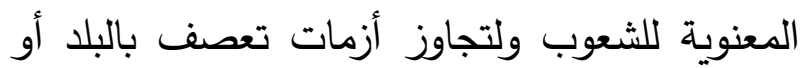
حتى لتعزيز الانتماء الوطني. فمثلاً قد كتب سوين (Swaine,2015) عن الصين الصنئ وكيف أن معظم المواطنين فيها فخورون جداً بتاريخهم الطويل كثقافة قوية وحيوية وككيان سياسي واجتماعي مؤثر للغاية وأنهم يعتقدون أن بلدهم وهيل تتنمي إلى الصفوف الأولى للقوى الكبرى في آسيا، وفي بعض النواحي على مستوى العالم أيضاً.

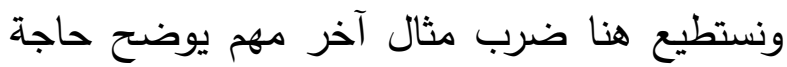
السلطة والمجتمع للتاريخ والتراث فالمهرجان الوطني للتراث والثقافة أو مهرجان الجنادرية (وهو مهرجان تراثي وثقافي يقام في المملكة العربية السعودية منذ عام .0ـ أهـ / 1910 (م) حيث أن من أسباب هذه الفعاليات هو إبراز الهوية الوطنية ولترسيخ المبادئ الذي قامت عليه هذه الدولة باستذكار المراحل التاريخية التي أدت لتكوّن هذا الكيان. ولو أخذنا جولة مختصرة على الدول العربية الأخرى

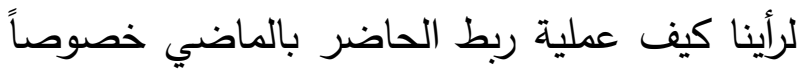
بالآثار وبالشخوص وبالكيانات التي كان لها تاريخ لهابخ مشرق وتعبر عن نهضة على هذه الأرض خلال الديموقراطية، تستخدم التاريخ لتكوين شعور بالهوية

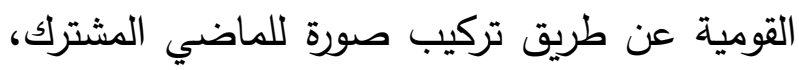

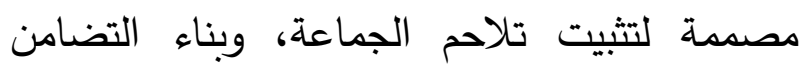
بينها (رينييه،19919 1، ص.9.9 (19). فصناعة الهوية القومية والوطنية من المهام العظيمة التي يقدمها التاريخ والمؤرخ لأي مجتمع ولذلك فهو يتغرد بها دون غيره.

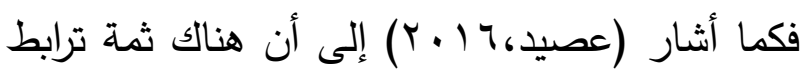
واضح وعميق ما بين مفاهيم الهوية والتاريخ والسياسة، وحيث تصبح الهوية شعوراً بالانتماء. التاريخ والمؤسسة السياسية والتأثير المتبادل يجب علينا قبل التحدث عن علاقة التاريخ بالمؤسسة السياسية تعريف هذه المؤسسة. فالمؤسسة السياسية الرسمية المعنية بصورة أكبر في لهوني هذا البحث هي المؤسسة الرسمية الفاعلة في نظام الحكم بسلطاتها الثلاث: السلطة القضائية، السلطة التشريعية كالبرلمان ومجلس الأمة والسلطة التنفيذية كمجس الوزراء. المؤسسات الرسمية التي يقيمها

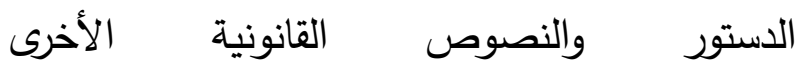
(دوفرجيه، ب999 19، صع 1) ). ولا يمكن تجاهل دور الأحزاب السياسية والقوى

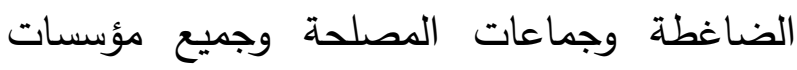
المجتمع المدني في التأثير بالمؤسسة السياسية 
شتاتهم من أوروبا والانتقال بادئ الأمر لإحدى المستعمرات في أفريقيا.

قد يزيد استخدام التاريخ والدعاية التاريخية من التان الأزمات التاريخية مع تغيير وتئزييف الحقائق

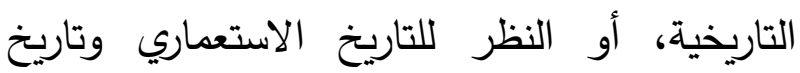
السيطرة على شعوب البلدان الأخرى. فاستخدام روسيا للتاريخ كسلاح سياسي قد يكون له له لهاء عواقب سلبية على المدى الطويل، ويمكن أن يؤدي لئحي لمواجهات أكثر مع الجيران والغرب، وتبقى الدول

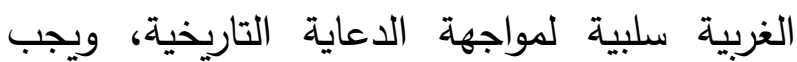
عليهم أن يعتمدوا على السرد القائم على الحقيقة

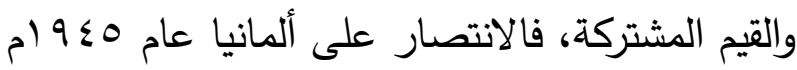
يأخذ مكانة تاريخية في ذاكرة روسيا التاريخية .(Prus,2015,P1)

ويبرز هنا استخدام الدعاية التاريخية في وقت الأزمات التي قد تتحول لصدام مسلح. وباضطلاع الاستجابات السياسية والقانونية للتاريخ المجدد بعملية التوضيح عند التعامل مع الذاكرة القومية، فمن الضروري التمييز بين التحليل التاريخي المشروع المبني على حقائق وبين التفسير التاريخي الذي يخدم برامج سياسية معاصرة. ومن الواضح أنه عندما تتحدد مناقشات التاريخ معاهرة. باتخ بالأصولية السياسية ينتج عن ذلك نوع من التاريخ

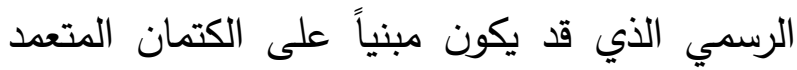

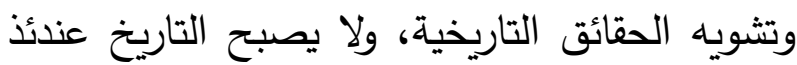

أزمنة مضت، فنرى استخدام مصر للتاريخ الفرعوني

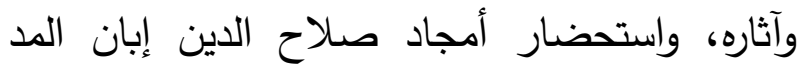
الناصري، وإقران دمشق بالأمويين لدى السورين للتذكير بعاصمة الخلافة القوية وتاريخ الملكة زنوبيا وبغداد الرشيد كما يحلو للعراقيين تسميتها وتاريخ نبوخذ نصر وحمورابي، واستخدامه من قبل نظام

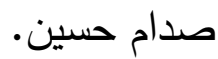
ويتضح لنا جلياً في العالم العربي التوجيه السياسي الصريح للتاريخ، وهذه من المعضلات الكبيرة التي لئي عقدت المشهد كثيراً.

إن استخدام التاريخ من قبل المؤسسة السياسية لترسيخ مبادئ الهوية الوطنية الجامعة، والعيش المشترك لمجتمع ما لا يقف عند هذا الحد بل يتعدى لتحسي لمحاولات تمرير أيديولوجيات هذا النظام الحاكم داخل المؤسسة السياسية ولبعث روح جديدة مستندة على تاريخ كان هناك قدم سبق لهذا الشعب أو ذاك كحاولات حزب العدالة والتتمية بالتذكير بالعهد

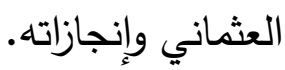
ليس توظيف التاريخ من قبل دولة أو كيان سياسي بمعزل عن الآخرين فقد يترتب هضم حقوق تاريخية

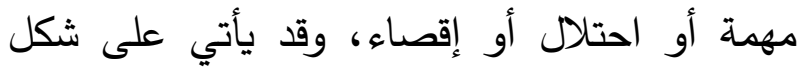
عدائي غير متصالح مع محيطه. فالكيان الصهيوني يتشدق على الدوم بالحق اليهودي بفلسطين علماً أنه من المعروف أن فلسطين كانت الخيار الثاني للحركة الصهيونية بعد أن أرادوا تجميع لهن لهين 
.(Appleby,Hunt,Jacub,1994,P290) والمثالان السابقان يعبران عن أول الخيط لبدأ عملية

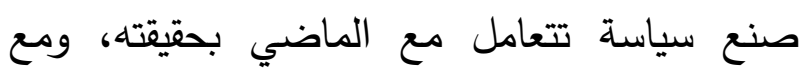
الحاضر وكل ذلك من أجل مستقبل موعود بالنجاح والاستقرار كما في تجارب دول عديدة كاليابان وألمانيا.

وهناك حالات قد يكون التاريخ سور منيع تستخدمه

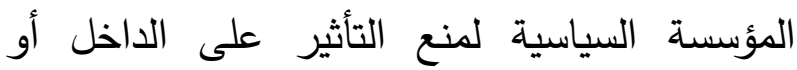
الاختراق ولتشكيل حصانة ضد ذلك وأيضاً كي تكون عملية صنع السياسة ناجعة بصورة كبيرة كما تم استخدامه من قبل الحكومات الصينية المتعاقبة وحتى على المستوى الثعبي داخلها. وترتبط تجربة الفوضى المحلية ارتباطاً وثيقاً بالنهب الذي تعرضت لله الصين من قبل القوى الغربية والإمبريالية واليابان في القرنين التاسع عشر وأوائل القرن العشرين ما يسمى بقرن الإذلال .(Swaine,2015,P1) فهنا قد أُستخدم التاريخ للتذكير بما تعرض له الوطن من العدوان والظلم ومن استعمار وسلب للثروات والمآسي التي عاشها هذا البلد في تلك الحقب يمنع وصول بعض الأفكار من خارج الحدود.

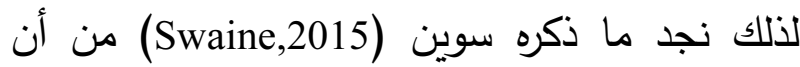
معظم الصينيين يقدر وجود حكومة وطنية قوية وموحدة ويقودها بفخر أفراد فاضلون يحافظون على وجلى ولى وطئ مصالح الناس، وبين أنهم في غالبهر لا يميلون
مصدراً، بل نتيجة للخيارات السياسية

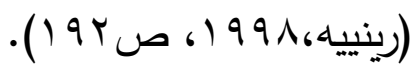
وتعريجاً على مسألة التلاعب بالتاريخ وتفاسيره فقد

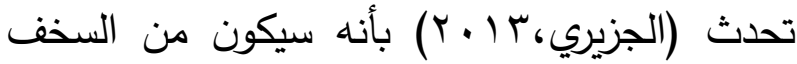

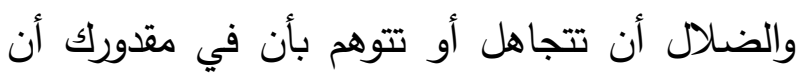
تتعامل مع التاريخ وتتجاهل روحه أو تعيد تشكيلها وصياغتها على هواك أو تفرض عليها سلطانك وتقرض عليها خصائص ليست فيها. التاريخ بين استخدام الحقائق وتوظيف التفاسير إن الكتابة التاريخية الصحيحة هي التي تكون مجردة وحرة وغير موجهة لتفسيرات معينة وإنما تكون لها نظرة بانورامية، وذات حيادية تتناول كل الجوانب المتاحة للاستفادة من كل التجارب الماضية الإيجابي منها السلبي، إذا كان بالفعل المراد من ذلك تطوير

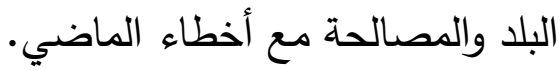
فعندما كسب المؤرخ الياباني سابورو إيناجا دعوى قضائية حكت بعدم شرعية رقابة وزارة التعليم اليابانية على المواد في كتب التاريخ المدرسية والمتعلقة ببشاعات اليابانيين في الحرب العالمية الثانية (رينييه،،1991 (19، صع 9 1). ونستطيع أيضاً ذكر مثال آخر على محاولات التهات المصالحة التاريخية، فحظر تدريس التاريخ الرسي التئي السابق، وإلغاء اختبارات تاريخ المدرسة الثانوية القومية،، عندما تحطم الإجماع الذي كان قائماً حول

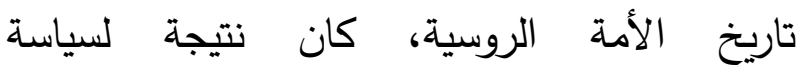
الجلاسنوست أو المكاشفة الانة 
إن المهارات والقدرة التحليلية لدى المؤرخين من أدوات المؤسسة الناجحة التي تجنبها تكرار أخطاء الماضي قدر الإمكان وتحاول استشراف ما سيكون عليه المستقبل عند استخدام بدائل عدة لقضية ما. ويعتبر (Weiss,1986,P31) أن نتائج البحث أو آراء

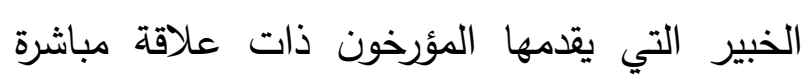

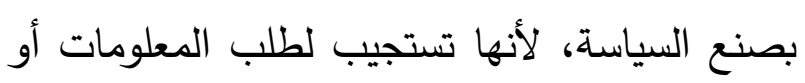

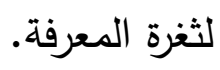

فليس إسهام المؤرخين في السياسة الخاصة ببعض

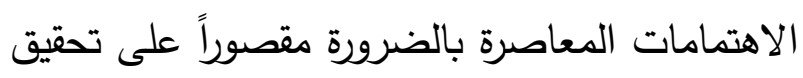
مشكلات معينة ذات أساس تاريخي، فهي قد تتشأ كذلك من تحقيق المؤرخ وبحثه الخاص والذي يلقي

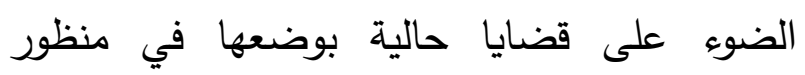

$$
\text { تاريخي. }
$$

وقد تطرق ويلكي (Wilkie,1974) لأهمية المعلومات

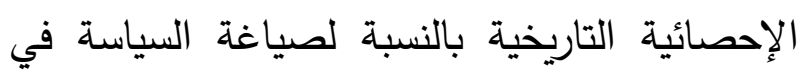
كل البلاد والتي تبرز التجارب الماضية كأساس لاستئس للتنبؤ بالاتجاهات المستقبلية المحتملة. ففي السياسات الداخلية التتموية نجد أن تحليل الإحصائيات التاريخية المتعلقة بالنمو السكاني في لهي مدينة مكسيكو سيتي التي تمت في السبعينات قد

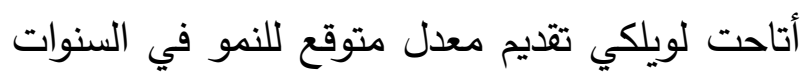

القادمة.

ويبين بيترسون (Peterson,1997) بأن الماضي مهر لأن النشاط السياسي السابق يشكل المؤسسات والقواعد التي تحكم إجراءاتها، ولأنه يغير معنى لأنى لمونيات
تاريخياً أو ثقافياً إلى تأييد نظام سياسي ديموقراطي غربي ليبرالي منقسم. دور التاريخ في صنع السياسة ليرل

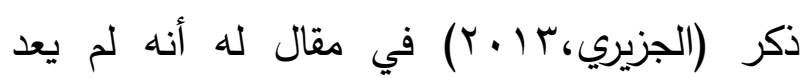
التاريخ عند المفكر الألماني هيجل مجرد سرد لوقائع الماضي التي انقضت، بل أصبح معنيا بالدرجة

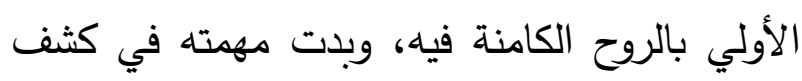
تجلياتها في حاضرنا. ويتبين للباحث إن حاجة المؤسسة السياسية للتاريخ ليست حاجة معنوية فقط بل تتجاوز إلى التأثير المباشر والإسهام في صنع السياسات الداخلية التتموية منها والأمنية والسياسات الخارجية والعلاقات الدولية. وقد ذكر (رينييه،1991، ص9911) أن التاريخ أداة في عملية صنع السياسة وهذا الدور ليس مفهوماً جديداً.

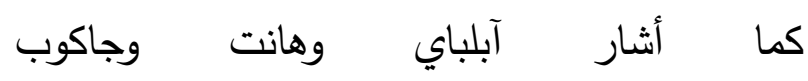
(Appleby,Hunt,Jacub, 1994) الجانب التاريخي تقع تحديات وصعوبات كان بالإمكان تجنبها، وأفضل ما يفعله المؤرخون هو تهوبات الربط بالماضي لإلقاء الضوء على على مشكلات الحاضر والمشكلات المحتملة في المستقبل البعيد والقريب، وهكذا يمكن توضيح أن معرفة المؤرخين

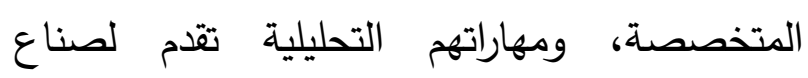
السياسة تفسيراً مهماً للتطور التاريخي. 
أنهم يقدمون منظوراً طويل الأجل ومتعلق بالسياسة العامة بشأن القضايا الدولية ويسهمون في المعرفة لاجنة

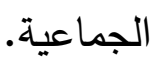

\section{الخاتمة}

تبين لنا من خلال ما استعرضناه في هذه الدراسة إن أي شعب لا يستطيع رسم هويته الخاصة دون هن هندين الاستناد للتاريخ فهو صانع الهوية، والجامع لأفراده وحلقة الربط بينه وبين تراثه، إضافة لرفع الروح

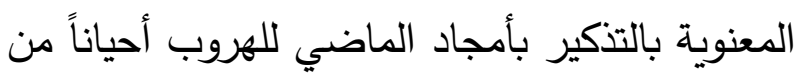

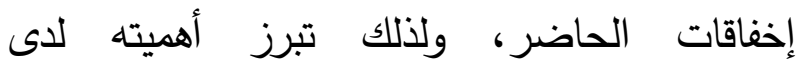
المؤسسات السياسية و التي يزداد اهتمامها بالتاريخ،

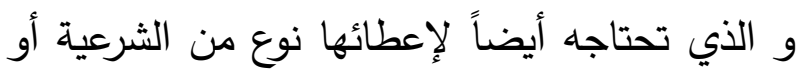

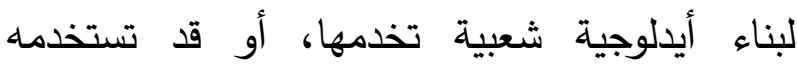
كسلاح لتصفية حسابات مع جهات أخرى، وفي الأخير قد يكون معولاً لهدم هذا الكيان أو ذاك، الكان ففقدان المصداقية وجعل المجتمع يعيش في أوهام أو لوان على الأقل على تفسيرات تاريخية محرفة تجعله في

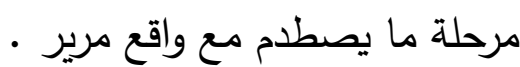
كما تبين للباحث أن الفائدة الحقيقية من التاريخ، ومن المؤرخين هي عندما يكون هناك مناخ عالي من الحرية وعدم محاولة فرض تفسيرات معينة

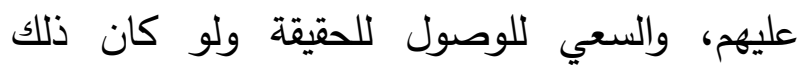

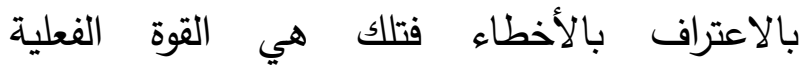
والانتصار الأخلاقي والتي على الاعتى المدى البعيد ستجنب الساسة والمجتمع من الوقوع بالأخطاء

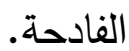

المصالح، وحدود السلطة في النظام السياسي، حيث

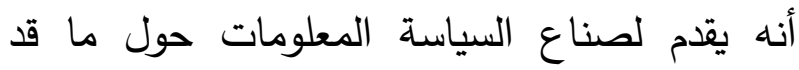
يحدث أو ما قد لا يحدث عندما تصاغ السياسات

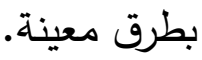
المؤرخ كسياسي ولا يُستغرب تحوّل المؤرخ من خبير يقدم دراسات

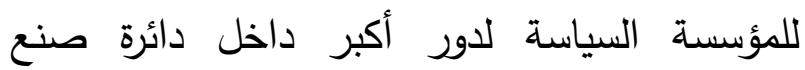
السياسة وفي حالات لصانع سياسة فعلي وكعضو لإن في هذه المؤسسة أو كوزير في الحكومة أو سفير لها. والأمثلة على دخول المؤرخ المعترك السياسي كثيرة، ففي القرن العشرين شكل المؤرخون الحكومة المحلية وجهوا الإمبراطوريات ونصحوا الرؤساء، فقد قاد المؤرخان فابيان بياتريس وسيدني ويب برنامج لندن، وهو خطة لتامين احتياجات لندن في مجال الإسكان والنقل والمياه، وذلك باستخدام الخبرة التي اكتسبوها في تأليف مجلدات تاريخية عن الحكومة منذ ودياه العصور الوسطى، كما عمل سيدني وييب كوزير

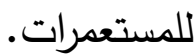
ولقد أصبح المؤرخ في جامعة هارفرد آرثر شليسنجر

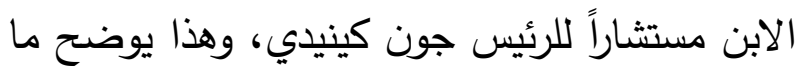
ذكر سلفاً من دور يتعاظم للمؤرخ داخل المؤسسة

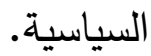

وقد سبق أن ذكر أرميتاغ (Armitage,2014) أن

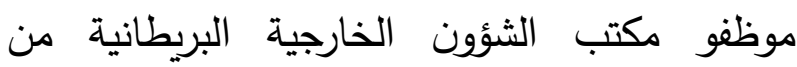
مؤرخين متفرغين أعلنوا على موقعهم على الانترنت 
قائمة المراجع المراجع العربية

لكتب:

ا ـ دوفرجيه، موري. (199r)."المؤسسات السياسية والثقانون اللستوري: الأنظمة السياسية لونية

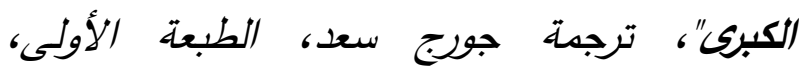

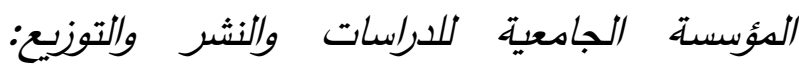

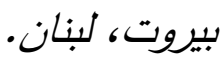

r. بينييه، روث. لبيل (1991). "التاريخ وصنع

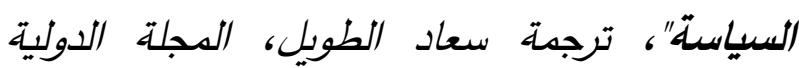

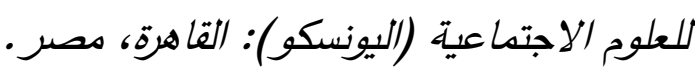

\section{Books:}

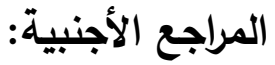

1. Appleby, J. Hunt, L. Jacub, M.1994."Telling the Truth about History". New York: Norton.

2. Barraclough, G. 1991. "Main Trends in History". New York: Holmes and Meier.

3. Hobsbawm, E. 1997. "On History". London: Weidenfeld and Nicolson.

4. Peterson, M.A. 1997. "The Limits of Social Learning: Translating Analysis into Action". Journal of Health Politics. Policy and law 22. 4, August, pp. 1077-1414.

http://www.ahram.org.eg/NewsQ/ $/$ \&.79) .aspx

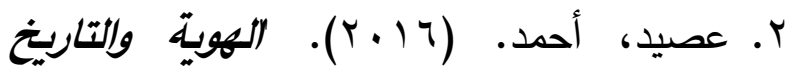
وإلسياسة، متابعات". مقال مقدم لموقع ناظور

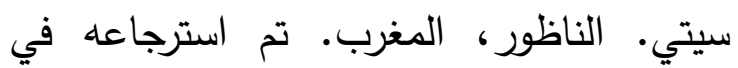

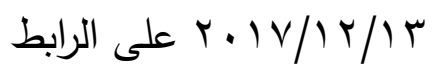

كما أن المنظور التاريخي، والقراءة التحليلية والاستتباطية إضافةً للعمليات الإحصائية من أدوات المؤرخين المساهمين في صنع السياسة الداخلية

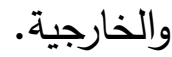
ويجرنا هذا على أنه يجب عند تدريب المؤرخين وبشكل أعم الطلبة الدارسين للتاريخ كمادة من ضمن لتهن المواد الأخرى، والذي قد يصبح بعضهم في المستقبل من صناع السياسة ضرورة زيادة التأكيد على المجال المنهجي المتاح للمؤرخ، وإمكانية تطبيق أساليب البحث العلمي مع الاهتمام بفهم كامل للمنظور

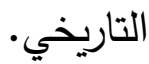

5. Weiss, C. 1986. "The Many Meanings of Research Utilization In Social Science and Social Policy". London. Allen and Unwin. 6. Wilkie, J.W. 1974. "Statistics and National Policy". Los Angeles: UCLA.

Journal Article II.

1. Clark, Anna. 2010. "Politicians Using History". Wiley-Blackwell, vol.56, no. 1, pp. 120-131.

2. Prus, Justyna. 2015. "Russia's Use of History as a Political Weapon". Policy Paper. The Polish Institute of International Affairs(PISM). No.12 (114), May 2015.

$$
\text { مصدر الكتروني: }
$$

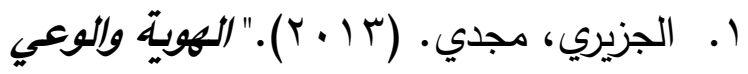

$$
\begin{aligned}
& \text { التاريخي عند هيجل". مقال مقدم لصحيفة الأهرام. }
\end{aligned}
$$

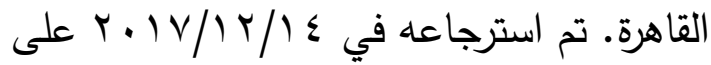

$$
\begin{aligned}
& \text { الرابط }
\end{aligned}
$$


https://www.theguardian.com/education/2014/ oct/07/why-politicians-need-historians

2. Swaine, Michael D. (2015)." China: The Influence of History". The Diplomat Magazine. Washington D.C. Retrieved 10/12/2017, https:/thediplomat.com/2015/01/china-theinfluence-of-history/ https://www.nadorcity.com-الهوية-و التاريخ _ a . T NV.html

\section{Electronic Media}

1. Armitage, David. (2014)." Why politicians need historians". The Guardian newspaper. London. $\quad$ Retrieved $9 / 12 / 2017$, 


\title{
History and Political Institution
}

\author{
Written by: Abdulhadi Naif Alsulami \\ Faculty of Arts and Humanities \\ Jeddah-Kingdom of Saudi Arabia
}

\begin{abstract}
. history is an important input in the political establishment, and in terms of the role played by that institution, where it is a resource used by the political institution towards the corresponding challenges, and the direction desired by it.

This study was to examine the relationship between history and politics and its role in the political establishment, and in the transformation of the historian from political scientist to political maker.

The researcher used the historical method for his suitability for this kind of studies. He concluded that history is used as a tool in the hands of the political establishment to derive its legitimacy and that the credibility of history is an important factor in preserving society under the umbrella of the political establishment. The freedom of historians to address history.

The researcher also stressed the importance of providing the methodological field available to the historian, with the need to apply the methods of scientific research, and to pay attention to the full understanding of the historical perspective of history learners.
\end{abstract}

